Technical note

\title{
Adjustment recommendations of a conoscopic holography sensor for a reliable scanning of surfaces with roughness grades obtained by different processes
}

\author{
J. Carlos Rico*, Gonzalo Valiño, Pedro Fernández, Pablo Zapico, David Blanco, \\ Sabino Mateos \\ Department of Construction and Manufacturing Engineering, University of Oviedo, Campus de Gijón, 33203 Gijón, Spain
}

\section{A R T I C L E I N F O}

\section{Article history:}

Received 28 November 2014

Received in revised form 13 April 2015

Accepted 20 April 2015

Available online 28 April 2015

\section{Keywords:}

Conoscopic holography

Non-contact scanning

Roughness

CMM

\begin{abstract}
A B S T R A C T
Conoscopic holography $(\mathrm{CH})$ is a non-contact interferometric technique used in surface digitizing. Like other laser techniques it is influenced by different factors such as surface reflectance, material, colour or even speckle noise caused by roughness. In this work, a $\mathrm{CH}$ system was used for analysing the influence of roughness on surface digitizing. For this purpose, several digitizing tests were performed on roughness specimens corresponding to EDM, face milling and flat grinding processes. Each roughness grade was digitized under different combinations of the sensor setting parameters (frequency $F$ and power $P$ ) which satisfy that the signal acquired by the sensor lies within the quality values recommended by the manufacturer. The results were analysed by using two indicators that show quality of the points captured by the sensor regarding the surface geometrical reconstruction and its metrological reliability. Finally, the study provides a series of recommendations for adjusting the sensor in order to satisfy both indicators simultaneously.
\end{abstract}

(c) 2015 Elsevier Inc. All rights reserved.

\section{Introduction}

Industrial use of commercial scanners like non-contact digitizing systems has grown significantly in recent years with a wide range of applications that go from dimensional metrology to reverse engineering [1-3]. Apart from avoiding contact with the object to be measured, the main advantages over contact systems are the ability to capture small geometries and complex shapes as well as the high speed for points acquisition. Additionally, the portability of non-contact systems offers the possibility to be installed on different equipment such as coordinate measuring machines (CMM), coordinate measuring arms, machine tools or production systems, which certainly favours its industrial application.

Despite the above advantages, commercial non-contact scanners are usually less accurate than the traditional contact-type methods, since their accuracy depends strongly on the relative position and orientation of the sensor with regard to the digitized part, the configuration parameters of the sensor, the part

\footnotetext{
* Corresponding author. Tel.: +34 985182062.

E-mail address: jcarlosr@uniovi.es (J.C. Rico).
}

geometry, the optical properties of material, the surface roughness, etc.

Currently, there exist numerous non-contact techniques for surface digitizing, such as those based on triangulation laser which are more deeply analysed and disseminated every day [4-8]. However, the performance of other technologies has not been fully described yet. This is the case of conoscopic holography $(\mathrm{CH})$.

$\mathrm{CH}$ is an interferometric technique based on the double refractive property of birefringent crystals. It was first described by Sirat and Psaltis [9] and patented by Optimet Optical Metrology LTD. When a polarized monochromatic light ray crosses the crystal, it is divided into two orthogonal polarizations, the ordinary and extraordinary rays, which travel at different speeds through the crystal. The speed of the ordinary ray is constant. However, the speed of the extraordinary ray depends on the angle of incidence. In order to make both rays interfere in the detector plane, two circular polarizers are placed before and after the crystal. The interference pattern obtained in the detector has a radial symmetry, so that all the information is contained in one radius. Therefore, given an appropriate calibration, it is possible to calculate the original distance to the light emitting point from the fundamental frequency of one of the signal rays. 